\title{
Existence and uniqueness of solutions for nonlinear implicit Caputo-Hadamard fractional differential equations with nonlocal conditions
}

\author{
Abdelouaheb Ardjounia ${ }^{a}$, Ahcene Djoudi ${ }^{b}$ \\ ${ }^{a}$ Department of Mathematics and Informatics, Souk Ahras University, P.O. Box 1553, Souk Ahras, Algeria. \\ ${ }^{b}$ Department of Mathematics, Annaba University, P.O. Box 12, Annaba, Algeria.
}

\begin{abstract}
In this paper, we use the Banach fixed point theorem to obtain the existence, interval of existence and uniqueness of solutions for nonlinear implicit Caputo-Hadamard fractional differential equations with nonlocal conditions. We also use the generalization of Gronwall's inequality to show the estimate of the solutions.

Keywords: Implicit fractional differential equations; Nonlocal conditions; Caputo-Hadamard fractional derivatives; Fixed point theorems; Existence; Uniqueness.

2010 MSC: 34A12, 34K20, 45N05.

\section{Introduction}

Fractional differential equations with and without delay arise from a variety of applications including in various fields of science and engineering such as applied sciences, practical problems concerning mechanics, the engineering technique fields, economy, control systems, physics, chemistry, biology, medicine, atomic energy, information theory, harmonic oscillator, nonlinear oscillations, conservative systems, stability and instability of geodesic on Riemannian manifolds, dynamics in Hamiltonian systems, etc. In particular, problems concerning qualitative analysis of linear and nonlinear fractional differential equations with and without delay have received the attention of many authors, see [1]-[25] and the references therein.
\end{abstract}

Email addresses: abd_ardjouni@yahoo.fr (Abdelouaheb Ardjouni), adjoudi@yahoo.com (Ahcene Djoudi) 
In [7, Dhaigude and Bhairat investigated the existence and stability of solutions of the following nonlinear implicit fractional differential equation

$$
\left\{\begin{array}{l}
\mathfrak{D}_{1}^{\alpha} x(t)=f\left(t, x(t), \mathfrak{D}_{1}^{\alpha} x(t)\right), t \in[1, b], b>1 \\
x^{(k)}(1)=x_{k} \in \mathbb{R}^{n}, k=0,1, \ldots, m-1
\end{array}\right.
$$

where $\mathfrak{D}_{1}^{\alpha}$ is the Caputo-Hadamard derivative of order $m-1<\alpha \leq m$. By employing the modified version of contraction principle and the successive approximation method, the authors obtained existence and stability results.

The implicit fractional differential equation

$$
\left\{\begin{array}{l}
{ }^{C} D^{\alpha} x(t)=f\left(t, x(t),{ }^{C} D^{\alpha} x(t)\right) \\
x(0)+g(x)=x_{0}
\end{array}\right.
$$

has been investigated in [11, where ${ }^{C} D^{\alpha}$ is the standard Caputo's fractional derivative of order $0<\alpha<1$. By using the contraction mapping principle, the existence, interval of existence and uniqueness of solutions has been established.

In this paper, we are interested in the analysis of qualitative theory of the problems of the existence, interval of existence and uniqueness of solutions to implicit Caputo-Hadamard fractional differential equations with nonlocal conditions. Inspired and motivated by the works mentioned above and the references in this paper, we concentrate on the existence, interval of existence and uniqueness of solutions for the nonlinear implicit Caputo-Hadamard fractional differential equation with nonlocal conditions

$$
\left\{\begin{array}{l}
\mathfrak{D}_{1}^{\alpha} x(t)=f\left(t, x(t), \mathfrak{D}_{1}^{\alpha} x(t)\right) \\
x(1)+g(x)=x_{0}
\end{array}\right.
$$

where $f:[1, T] \times \mathbb{R}^{2} \rightarrow \mathbb{R}$ and $g: C([1, T], \mathbb{R}) \rightarrow \mathbb{R}$ are nonlinear continuous functions and $\mathfrak{D}_{1}^{\alpha}$ denotes the Caputo-Hadamard derivative of order $0<\alpha<1$. In passing, we note that the application of nonlinear condition $x(1)+g(x)=x_{0}$ in physical problems yields better effect than the initial condition $x(1)=x_{0}$ (see [4]). To show the existence, interval of existence and uniqueness of solutions, we transform (11) into an integral equation and then use the Banach fixed point theorem. Further, by the generalization of Gronwall's inequality we obtain the estimate of solutions of (1).

\section{Preliminaries}

In this section we present some basic definitions, notations and results of fractional calculus [1, 8, 10, 15, 16, 21] which are used throughout this paper.

Definition 2.1 ([16]). The Hadamard fractional integral of order $\alpha>0$ for a continuous function $x$ : $[1,+\infty) \rightarrow \mathbb{R}$ is defined as

$$
\mathfrak{I}_{1}^{\alpha} x(t)=\frac{1}{\Gamma(\alpha)} \int_{1}^{t}\left(\log \frac{t}{s}\right)^{\alpha-1} x(s) \frac{d s}{s}, \alpha>0
$$

Definition 2.2 ([1, 10, 15]). The Caputo-Hadamard fractional derivative of order $\alpha$ for a continuous function $x:[1,+\infty) \rightarrow \mathbb{R}$ is defined as

$$
\mathfrak{D}_{1}^{\alpha} x(t)=\frac{1}{\Gamma(n-\alpha)} \int_{1}^{t}\left(\log \frac{t}{s}\right)^{n-\alpha-1} \delta^{n}(x)(s) \frac{d s}{s}, n-1<\alpha<n,
$$

where $\delta^{n}=\left(t \frac{d}{d t}\right)^{n}, n=[\alpha]+1$. 
Lemma $2.3([15])$. Let $\Re(\alpha)>0$. Suppose $x \in C^{n-1}[1,+\infty)$ and $\delta^{(n)} x(t)$ exists almost everywhere on any bounded interval of $[1,+\infty)$. Then

$$
\mathfrak{I}_{1}^{\alpha}\left[\mathfrak{D}_{1}^{\alpha} x(t)\right]=x(t)-\sum_{k=0}^{n-1} \frac{\delta^{k)} x(1)}{\Gamma(k+1)}(\log t)^{k} .
$$

In particular, when $0<\Re(\alpha)<1, \mathfrak{I}_{1}^{\alpha}\left[\mathfrak{D}_{1}^{\alpha} x(t)\right]=x(t)-x(1)$.

Lemma 2.4 ([16]). For all $\mu>0$ and $\nu>-1$, then

$$
\frac{1}{\Gamma(\mu)} \int_{1}^{t}\left(\log \frac{t}{s}\right)^{\mu-1}(\log s)^{\nu} \frac{d s}{s}=\frac{\Gamma(\nu+1)}{\Gamma(\mu+\nu+1)}(\log t)^{\mu+\nu} .
$$

The following generalization of Gronwall's lemma for singular kernels plays an important role in obtaining our main results.

Lemma $2.5([20])$. Let $x:[1, T] \rightarrow[0, \infty)$ be a real function and $w$ is a nonnegative locally integrable function on $[1, T]$. Assume that there is a constant $a>0$ such that for $0<\alpha<1$

$$
x(t) \leq w(t)+a \int_{1}^{t}\left(\log \frac{t}{s}\right)^{\alpha-1} x(s) \frac{d s}{s} .
$$

Then, there exist a constant $K=K(\alpha)$ such that

$$
x(t) \leq w(t)+K a \int_{1}^{t}\left(\log \frac{t}{s}\right)^{\alpha-1} w(s) \frac{d s}{s},
$$

for every $t \in[1, T]$.

\section{Main results}

In this section, we give the equivalence of the initial value problem (1) and prove the existence, interval of existence, uniqueness and estimate of solutions of (1).

The proof of the following lemma is close to the proof of Lemma 6.2 given in [8].

Lemma 3.1. If the functions $f:[1, T] \times \mathbb{R}^{2} \rightarrow \mathbb{R}$ and $g: C([1, T], \mathbb{R}) \rightarrow \mathbb{R}$ are continuous, then the initial value problem (1) is equivalent to nonlinear fractional Volterra integro-differential equation

$$
x(t)=x_{0}-g(x)+\frac{1}{\Gamma(\alpha)} \int_{1}^{t}\left(\log \frac{t}{s}\right)^{\alpha-1} f\left(s, x(s), \mathfrak{D}_{1}^{\alpha} x(s)\right) \frac{d s}{s}, t \in[1, T] .
$$

Theorem 3.2. Let $T>1$. Assume $f:[1, T] \times \mathbb{R}^{2} \rightarrow \mathbb{R}$ and $g: C([1, T], \mathbb{R}) \rightarrow \mathbb{R}$ satisfy the following condition

(H1) There exist $K_{1} \in \mathbb{R}^{+}, K_{2}, K_{3} \in(0,1)$ such that

$$
|f(t, u, v)-f(t, \tilde{u}, \tilde{v})| \leq K_{1}|u-\tilde{u}|+K_{2}|v-\tilde{v}|,
$$

and

$$
|g(x)-g(\tilde{x})| \leq K_{3}\|x-\tilde{x}\| .
$$

Let

$$
1<b<\min \left\{T, \exp \left(\frac{\left(1-K_{3}\right)\left(1-K_{2}\right) \Gamma(\alpha+1)}{K_{1}}\right)^{\frac{1}{\alpha}}\right\}
$$

then (1) has a unique solution $x \in C([1, b], \mathbb{R})$. 
Proof. Let

$$
\mathfrak{D}_{1}^{\alpha} x(t)=z_{x}(t), x(1)+g(x)=x_{0},
$$

then by Lemma 3.1 .

$$
x(t)=x_{0}-g(x)+\frac{1}{\Gamma(\alpha)} \int_{1}^{t}\left(\log \frac{t}{s}\right)^{\alpha-1} z_{x}(s) \frac{d s}{s}, t \in[1, T]
$$

where

$$
z_{x}(t)=f\left(t, x_{0}-g(x)+\mathfrak{I}_{1}^{\alpha} z_{x}(t), z_{x}(t)\right) .
$$

That is $x(t)=x_{0}-g(x)+\mathfrak{I}_{1}^{\alpha} z_{x}(t)$. Define the mapping $P: C([1, b], \mathbb{R}) \rightarrow C([1, b], \mathbb{R})$ as follows

$$
(P x)(t)=x_{0}-g(x)+\frac{1}{\Gamma(\alpha)} \int_{1}^{t}\left(\log \frac{t}{s}\right)^{\alpha-1} z_{x}(s) \frac{d s}{s} .
$$

It is clear that the fixed points of $P$ are solutions of $[1]$. Let $x, y \in C([1, b], \mathbb{R})$, then we have

$$
\begin{aligned}
& |(P x)(t)-(P y)(t)| \\
& \leq|g(x)-g(y)|+\frac{1}{\Gamma(\alpha)} \int_{1}^{t}\left(\log \frac{t}{s}\right)^{\alpha-1}\left|z_{x}(s)-z_{y}(s)\right| \frac{d s}{s} \\
& \leq K_{3}\|x-y\|+\frac{1}{\Gamma(\alpha)} \int_{1}^{t}\left(\log \frac{t}{s}\right)^{\alpha-1}\left|z_{x}(s)-z_{y}(s)\right| \frac{d s}{s}
\end{aligned}
$$

and

$$
\begin{aligned}
\left|z_{x}(t)-z_{y}(t)\right| & \leq\left|f\left(t, x(t), z_{x}(t)\right)-f\left(t, x(t), z_{y}(t)\right)\right| \\
& \leq K_{1}|x(t)-y(t)|+K_{2}\left|z_{x}(t)-z_{y}(t)\right| \\
& \leq \frac{K_{1}}{1-K_{2}}|x(t)-y(t)| .
\end{aligned}
$$

By replacing (5) in the inequality (4), we get

$$
\begin{aligned}
& |(P x)(t)-(P y)(t)| \\
& \leq K_{3}\|x-y\|+\frac{1}{\Gamma(\alpha)} \frac{K_{1}}{1-K_{2}} \int_{1}^{t}\left(\log \frac{t}{s}\right)^{\alpha-1}|x(s)-y(s)| \frac{d s}{s} \\
& \leq K_{3}\|x-y\|+\frac{1}{\Gamma(\alpha)} \frac{K_{1}}{1-K_{2}}\left(\int_{1}^{t}\left(\log \frac{t}{s}\right)^{\alpha-1} \frac{d s}{s}\right)\|x-y\| \\
& \leq\left(K_{3}+\frac{K_{1}}{1-K_{2}} \frac{(\log t)^{\alpha}}{\Gamma(\alpha+1)}\right)\|x-y\| .
\end{aligned}
$$

Since $t \in[1, b]$, then

$$
\|P x-P y\| \leq \beta\|x-y\|, 0<\beta<1,
$$

where

$$
\beta=K_{3}+\frac{K_{1}}{1-K_{2}} \frac{(\log b)^{\alpha}}{\Gamma(\alpha+1)} .
$$

That is to say the mapping $P$ is a contraction in $C([1, b], \mathbb{R})$. Hence, by the Banach fixed point theorem, $P$ has a unique fixed point $x \in C([1, b], \mathbb{R})$. Therefore, (1) has a unique solution. 
Theorem 3.3. Assume that $f:[1, T] \times \mathbb{R}^{2} \rightarrow \mathbb{R}$ and $g: C([1, T], \mathbb{R}) \rightarrow \mathbb{R}$ satisfy (H1). If $x$ is a solution of (1), then

$$
\begin{aligned}
\|x\| \leq & \frac{\left(1-K_{2}\right)\left(1-K_{3}\right) \Gamma(\alpha+1)+K_{1} K(\log T)^{\alpha}}{\left(1-K_{2}\right)\left(1-K_{3}\right)^{2} \Gamma(\alpha+1)} \\
& \times\left(\left|x_{0}\right|+Q_{1}+\frac{Q_{2}(\log T)^{\alpha}}{\left(1-K_{2}\right) \Gamma(\alpha+1)}\right),
\end{aligned}
$$

where $Q_{1}=|g(0)|, Q_{2}=\sup _{t \in[1, T]}|f(t, 0,0)|$ and $K \in \mathbb{R}^{+}$is a constant.

Proof. Let

$$
\mathfrak{D}_{1}^{\alpha} x(t)=z_{x}(t), x(1)+g(x)=x_{0} .
$$

By Lemma 3.1. $x(t)=x_{0}-g(x)+\mathfrak{I}_{1}^{\alpha} z_{x}(t)$. Then by hypothesis (H1), for any $t \in[1, T]$ we have

$$
\begin{aligned}
|x(t)| & \leq\left|x_{0}\right|+|g(x)|+\mathfrak{I}_{1}^{\alpha}\left|z_{x}(t)\right| \\
& \leq\left|x_{0}\right|+|g(x)-g(0)|+|g(0)|+\mathfrak{I}_{1}^{\alpha}\left|z_{x}(t)\right| \\
& \leq\left|x_{0}\right|+Q_{1}+K_{3}\|x\|+\mathfrak{I}_{1}^{\alpha}\left|z_{x}(t)\right|,
\end{aligned}
$$

where $Q_{1}=|g(0)|$. On the other hand, for any $t \in[1, T]$ we get

$$
\begin{aligned}
\left|z_{x}(t)\right| & =\left|f\left(t, x(t), z_{x}(t)\right)\right| \\
& \leq\left|f\left(t, x(t), z_{x}(t)\right)-f(t, 0,0)\right|+|f(t, 0,0)| \\
& \leq K_{1}|x(t)|+K_{2}\left|z_{x}(t)\right|+|f(t, 0,0)| \\
& \leq \frac{K_{1}}{1-K_{2}}\|x\|+\frac{Q_{2}}{1-K_{2}},
\end{aligned}
$$

where $Q_{2}=\sup _{t \in[1, T]}|f(t, 0,0)|$. Therefore

$$
|x(t)| \leq\left|x_{0}\right|+Q_{1}+K_{3}\|x\|+\mathfrak{I}_{1}^{\alpha}\left(\frac{Q_{2}}{1-K_{2}}+\frac{K_{1}}{1-K_{2}}\|x\|\right) .
$$

Thus

$$
\begin{aligned}
\left(1-K_{3}\right)\|x\| \leq & \left|x_{0}\right|+Q_{1}+\frac{Q_{2}(\log T)^{\alpha}}{\left(1-K_{2}\right) \Gamma(\alpha+1)} \\
& +\frac{K_{1}}{\left(1-K_{2}\right)\left(1-K_{3}\right)} \mathfrak{I}_{1}^{\alpha}\left\{\left(1-K_{3}\right)\|x\|\right\} .
\end{aligned}
$$

By Lemma 2.5, there is a constant $K=K(\alpha)$ such that

$$
\begin{aligned}
& \left(1-K_{3}\right)\|x\| \\
& \leq\left|x_{0}\right|+Q_{1}+\frac{Q_{2}(\log T)^{\alpha}}{\left(1-K_{2}\right) \Gamma(\alpha+1)} \\
& +\frac{K_{1} K(\log T)^{\alpha}}{\left(1-K_{2}\right)\left(1-K_{3}\right) \Gamma(\alpha+1)}\left(\left|x_{0}\right|+Q_{1}+\frac{Q_{2}(\log T)^{\alpha}}{\left(1-K_{2}\right) \Gamma(\alpha+1)}\right) \\
& \leq \frac{\left(1-K_{2}\right)\left(1-K_{3}\right) \Gamma(\alpha+1)+K_{1} K(\log T)^{\alpha}}{\left(1-K_{2}\right)\left(1-K_{3}\right) \Gamma(\alpha+1)} \\
& \times\left(\left|x_{0}\right|+Q_{1}+\frac{Q_{2}(\log T)^{\alpha}}{\left(1-K_{2}\right) \Gamma(\alpha+1)}\right) .
\end{aligned}
$$


Hence

$$
\begin{aligned}
\|x\| \leq & \frac{\left(1-K_{2}\right)\left(1-K_{3}\right) \Gamma(\alpha+1)+K_{1} K(\log T)^{\alpha}}{\left(1-K_{2}\right)\left(1-K_{3}\right)^{2} \Gamma(\alpha+1)} \\
& \times\left(\left|x_{0}\right|+Q_{1}+\frac{Q_{2}(\log T)^{\alpha}}{\left(1-K_{2}\right) \Gamma(\alpha+1)}\right) .
\end{aligned}
$$

This completes the proof.

Acknowledgements. The authors would like to thank the anonymous referee for his valuable comments.

\section{References}

[1] Y. Adjabi, F. Jarad, D. Baleanu, T. Abdeljawad, On Cauchy problems with Caputo Hadamard fractional derivatives, Journal of Computational Analysis and Applications 21(1) (2016) 661-681.

[2] R.P. Agarwal, Y. Zhou, Y. He, Existence of fractional functional differential equations, Computers and Mathematics with Applications 59 (2010) 1095-1100.

[3] Z. Ali, A. Zada, K. Shah, On Ulam's stability for a coupled systems of nonlinear implicit fractional differential equations, Bull. Malays. Math. Sci. Soc. DOI.org/10.1007/s40840-018-0625-x.

[4] A. Bashir, S. Sivasundaram, Some existence results for fractional integro-differential equations with nonlocal conditions, Communications in Applied Analysis 12 (2008) 107-112.

[5] H. Boulares, A. Ardjouni, Y. Laskri, Positive solutions for nonlinear fractional differential equations, Positivity 21 (2017) 1201-1212.

[6] H. Boulares, A. Ardjouni, Y. Laskri, Stability in delay nonlinear fractional differential equations, Rend. Circ. Mat. Palermo 65 (2016) 243-253.

[7] D.B. Dhaigude, S.P. Bhairat, On Ulam type stability for nonlinear implicit fractional differential equations, arXiv: 1707.07597v1, [math.CA] 24 Jul 2017.

[8] K. Diethelm, The analysis of fractional differential equations, Lecture Notes in Mathematics, Springer-verlag, Berlin, Heidelberg (2010).

[9] J. Dong, Y. Feng and J. Jiang, A note on implicit fractional differential equations, Mathematica Aeterna 7(3) (2017) 261-267.

[10] Y. Gambo, F. Jarad, D. Baleanu, F. Jarad, On Caputo modification of the Hadamard fractional derivatives, Advances in Difference Equations 2014(10) (2014) 1-12.

[11] M. Haoues, A. Ardjouni and A. Djoudi, Existence, interval of existence and uniqueness of solutions for nonlinear implicit Caputo fractional differential equations, TJMM 10(1) (2018) 09-13.

[12] S. Harikrishnan, K. Shah, D. Baleanu, K. Kanagarajan, Note on the solution of random differential equations via-Hilfer fractional derivative, Advances in Difference Equations 2018(224) (2018) 1-9.

[13] F. Jarad, T. Abdeljawad, Generalized fractional derivatives, and Laplace transform, Discrete and Continuous dynamic systems DOI:10.3934/dcdss.2020039.

[14] F. Jarad, T. Abdeljawad, D. Baleanu, On the generalized fractional derivatives and their Caputo modification, Journal of Nonlinear Sciences and Applications 10(5) (2017) 2607-2619.

[15] F. Jarad, T. Abdeljawad, D. Baleanu, Caputo-type modification of the Hadamard fractional derivatives, Advances in Difference Equations 2012(142) (2012) 1-8.

[16] A.A. Kilbas, H.M. Srivastava and J. J. Trujillo, Theory and Applications of Fractional Differential Equations, North-Holland Mathematics Studies 204, Editor: Jan Van Mill, Elsevier, Amsterdam, The Netherlands (2006).

[17] K.D. Kucche, J.J. Nieto and V. Venktesh, Theory of nonlinear implicit fractional differential equations, Differ. Equ. Dyn. Syst. DOI 10.1007/s12591-016-0297-7.

[18] K.D. Kucche, S.T. Sutar, On existence and stability results for nonlinear fractional delay differential equations, Bol. Soc. Paran. Mat. (3s.) v. 36 (4) (2018) 55-75.

[19] K.D. Kucche, S.S. Sutar, Stability via successive approximation for nonlinear implicit fractional differential equations, Moroccan J. Pure Appl. Anal. 3(1) (2017) 36-55.

[20] S.-Y. Lin, Generalized Gronwall inequalities and their applications to fractional differential equations, Journal of Inequalities and Applications 2013(549) (2013) 1-9.

[21] I. Podlubny, Fractional differential equations, Academic Press, San Diego (1999).

[22] K. Shah, R. Ali Khan, Iterative scheme for a coupled system of fractional-order differential equations with three-point boundary conditions, Mathematical Methods in the Applied Sciences 41(3) (2018) 1047-1053.

[23] S. T. Sutar, K. D. Kucche, Global existence and uniqueness for implicit differential equations of arbitrary order, Fractional Differential Calculus 5(2) (2015) 199-208.

[24] J. Wang, L. Lv, Y. Zhou, New concepts and results in stability of fractional differential equations, Commun Nonlinear Sci Numer Simulat 17 (2012) 2530-2538. 
[25] J.R. Wang, K. Shah, A. Ali, Existence and Hyers-Ulam stability of fractional nonlinear impulsive switched coupled evolution equations, Mathematical Methods in the Applied Sciences 41(6) (2018) 2392-2402. 